\title{
Magnetic Moments at the Surface of Antiferromagnetic NiO(100)
}

\author{
F. U. Hillebrecht,* H. Ohldag, N. B. Weber, C. Bethke, and U. Mick \\ Institut für Angewandte Physik, Universität Düsseldorf, Universitätsstrasse 1, 40225 Düsseldorf, Germany
}

M. Weiss and J. Bahrdt

BESSY GmbH, Albert-Einstein-Strasse 15, 12489 Berlin, Germany

(Received 21 March 2000)

\begin{abstract}
The orientation of magnetic moments at the (100) surface of antiferromagnetic NiO single crystals is studied by $\mathrm{x}$-ray linear magnetic dichroism in photoemission microscopy. $T$ domains are observed terminating at the surface, with domain boundaries running mostly along in-plane [10] directions. From the detailed polarization dependence we find that the magnetic surface structure of a cleaved crystal is bulk terminated. This is in contrast to sputtered surfaces, where magnetic moments lie within the surface plane, forming a magnetically relaxed structure. These findings are of importance for understanding the exchange bias phenomenon.
\end{abstract}

DOI: $10.1103 /$ PhysRevLett.86.3419

PACS numbers: 78.70.Dm, 79.60.Bm

For any magnetic material the break of symmetry and reduced coordination at the surface may cause a deviation of the magnetic state at the surface from a bulk termination. Phenomena of this type have been reported for ferromagnetic surfaces, but they may occur equally well for antiferromagnetic (AF) surfaces. The influence of the surface may be manifest in a change of the Néel temperature, the magnitude and orientation of the magnetic moment, or even in a change of the antiferromagnetic structure. In the light of the interesting physical phenomena and also their technical relevance, e.g., in the context of spin electronics [1] and exchange bias [2], our knowledge about the magnetic structure of AF surfaces and interfaces is very limited. Since there is no magnetostatic energy in an antiferromagnet, even the origin of domain formation is a subject of debate. Experimental techniques which establish the AF ground state as such [3-9] can in principle provide information on the details of the magnetic structure, but there are only a few surface sensitive techniques [10-13]. In this Letter we use polarization dependent $\mathrm{x}$-ray absorption $[14,15]$ with spatial resolution $[16,17]$ to image AF domains and to determine the orientation of magnetic moments on a $\mathrm{NiO}(100)$ single crystal surface from the polarization dependence. For cleaved surfaces, the observations are consistent with the bulk magnetic structure. For rough surfaces, our data show a deviation of the surface magnetic structure from a bulk termination, i.e., a magnetic surface relaxation.

$\mathrm{NiO}$ is a collinear antiferromagnet with ferromagnetic (111) sheets, stacked in an antiferromagnetic order [3-5]. The possible bulk domains are listed in Table I. Domains associated with the four different 111 sheets are labeled $T_{1}$ through $T_{4}$. For any $T$ domain $T_{i}$, the spins lie within the associated 111 plane, pointing in any of the three [211]-derived directions as listed in Table I. Portions of a crystal with different spin directions are termed $S$ domains, which we denote by a second subscript $T_{i j}$ $(j=1-3)$.
To obtain a spatially resolved measure for the x-ray absorption coefficient, we measure the local electron yield, which is proportional to the local absorption coefficient [18], in a photoemission electron microscope (PEEM). In such a microscope, electrons excited from the sample are transferred by an electron-optical system to generate an enlarged map of the emission probability on a channel plate with fluorescent screen [19]. Contrast arises from a variation of the absorption coefficient connected to magnetic linear dichroism. For the large field of view used here the resolution was about $1 \mu \mathrm{m}$. For excitation we used synchrotron radiation generated by the elliptical undulator UE $56 / 1$ at the new BESSY II storage ring in Berlin [20] or from BW3 at HASYLAB in Hamburg. NiO crystals were cleaved either in situ in UHV or ex situ immediately before insertion into the microscope yielding consistent results. In some cases surfaces were sputtered by $500 \mathrm{eV}$ Ar ions before imaging. Scanning tunneling microscopy images of sputtered $\mathrm{NiO}(100)$ showed large flat terraces 1-2 $\mu \mathrm{m}$ wide, separated by steps about $1 \mathrm{~nm}$ high. Spin-resolved inelastic electron scattering [21] on such a $\mathrm{NiO}$ surface shows all spectral features related to bulk transitions in agreement with data for cleaved surfaces; surface loss features are still weakly present with this preparation.

The x-ray absorption spectrum of $\mathrm{NiO}$ shows rich structures which arise from the electrostatic interactions (Coulomb and exchange) between core hole and localized valence electrons with spin and orbital momentum. The angular dependent part of the spectrum is given by $\left(3 \cos ^{2} \theta-1\right) I^{(2)}$ where $\theta$ is the angle between magnetic

TABLE I. Bulk antiferromagnetic domains in NiO.

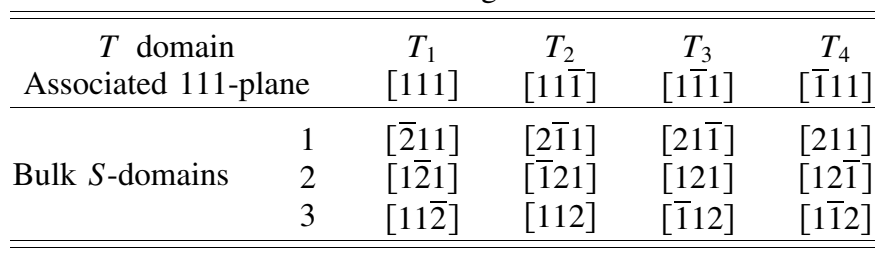

(C) 2001 The American Physical Society

3419 
moment and light polarization. $I^{(2)}$ describes its spectral shape [15]. For $\mathrm{NiO}$ the angular dependence is best detected in the doublet structure of the $2 p_{1 / 2}$ absorption edge [15] occurring at photon energies of 871.2 and $872.2 \mathrm{eV}$.

Figure 1 shows an image of the asymmetry $A=$ $\left(I_{1}-I_{2}\right) /\left(I_{1}+I_{2}\right)$, where $I_{1}(x, y)$ and $I_{2}(x, y)$ are intensity maps obtained for the two photon energies of the Ni $2 p_{1 / 2}$ absorption feature of $\mathrm{NiO}$ [22]. The bright and dark areas on the image indicate smaller and larger angles between magnetic moments and light polarization. This demonstrates the presence of different AF domains at the surface. The domain boundaries run along [001] directions in the surface. The widths of the domains are a few tens of $\mu \mathrm{m}$, and they extend over much larger distances. The domains are not affected by major defects on the surface, which shows that their topology at the surface is largely determined by the bulk domain structure. The $\mathrm{NiO} 2 p_{1 / 2}$ absorption spectrum derived from a series of images shows the well-known shape with two peaks at 871.2 and $872.2 \mathrm{eV}$ photon energy [15]. The magnetic dichroism in these two features amounts to 5.1 and $-1.6 \%$, respectively.

To further corroborate this finding, we show in Fig. 2 the temperature dependence of the magnetic contrast. The data show an essentially parabolic temperature dependence, and show surface ordering within experimental uncertainty at the bulk Néel temperature. This result is in good agreement with the temperature dependence of magnetic superstructure reflections in LEED [23]. Both experiments yield a signal proportional to the square of the AF order parameter. On cooling down, the domain pattern reappears without changes below the Néel temperature. The unchanged domain pattern suggests that size and topology of the domains

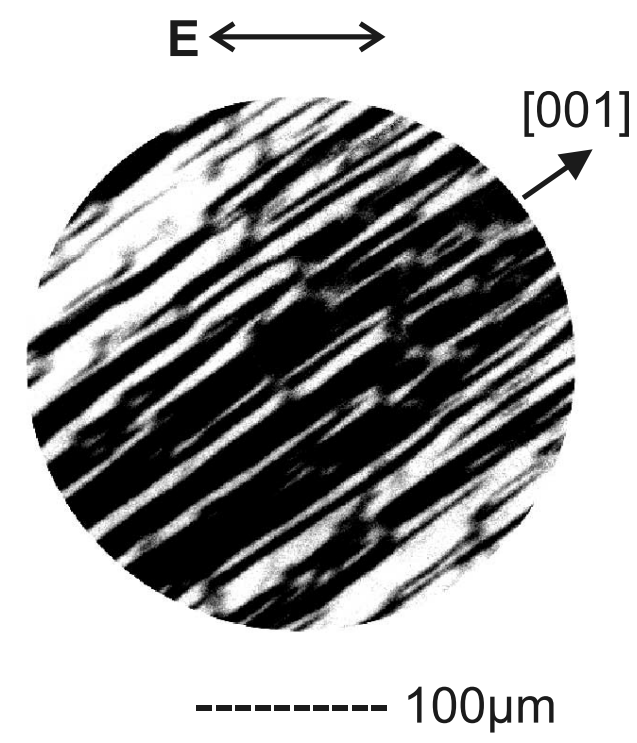

FIG. 1. AF domains on a cleaved $\mathrm{NiO}(100)$ surface. Doubleheaded arrow shows light polarization, and arrow at image indicates the [001] direction in the surface plane. The field of view is $300 \mu \mathrm{m}$. are largely governed by defects and stresses which are incorporated in the crystal probably already during growth.

To characterize the orientation of the magnetic moments at the surface, we study the contrast between AF domains as a function of light polarization. By rotating the sample azimuthally under the microscope, the angle dependent part of the absorption spectrum changes differently for different domains, leading to a change of the contrast. Figure 3 shows the expected variation with azimuthal sample orientation with respect to the electric field as given by the polarization dependence $\left(3 \cos ^{2} \theta-1\right)$, assuming bulk termination of the magnetic structure. Since there are 12 AF domains, one has 12 curves each for $s$ and $p$ polarization. For $s$ polarization, the 12 domains form six pairs with the same angular dependence, three of which are shown in Fig. 3a. The difference between any of the curves is a measure for the AF contrast between these domains. For obliquely incident $p$-polarized light, the contrast varies in a different fashion, as shown in Fig. 3b. From the azimuthal orientations for which the AF contrast disappears, or where it has a maximum, we identify which domains are present.

Figure 4 shows the experimental variation of the contrast between different AF domains with azimuthal orientation, determined from a series of asymmetry images excited with $s$-polarized light. Figure 4a shows that the contrast disappears at angles $90^{\circ}$ apart. At those positions where the contrast vanishes, the light polarization is parallel to a [001] direction within the surface plane. As shown in Fig. 3a, the variation of the angular dependent part is smaller for $T_{i 1}$ domains than for $T_{i 2}$ (and $T_{i 3}$ ) and the associated curves cross each other at angles of $-90^{\circ},-57^{\circ}$, $25^{\circ}, 90^{\circ}$, etc., i.e., for orientations separated by angles different from $90^{\circ}$. Therefore the domains can be related to either $T_{i 1}$ (large component of moment perpendicular to surface) or to $T_{i 2}$ and/or $T_{i 3}$ domains (large in-plane component of magnetic moment). The maximum contrast

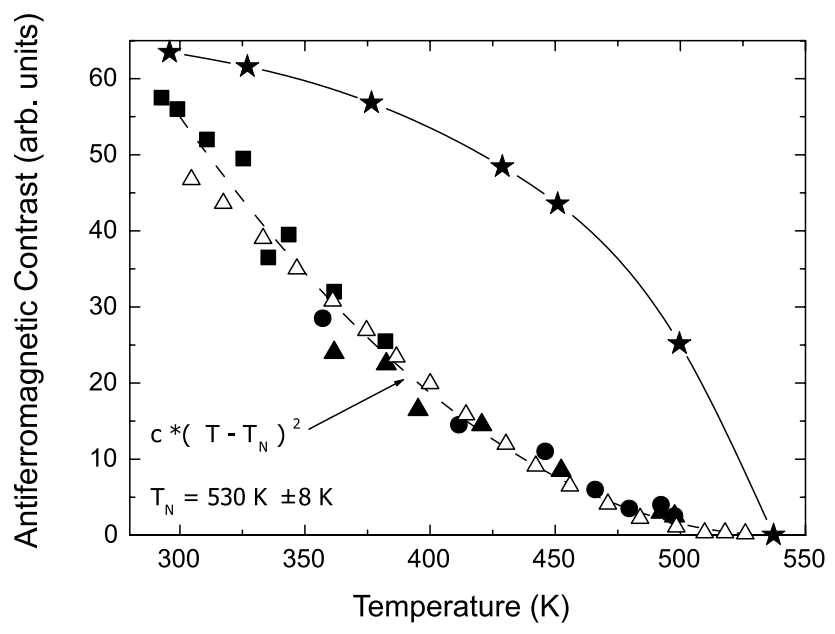

FIG. 2. Contrast between $\mathrm{NiO}$ antiferromagnetic domains as a function of temperature, derived from AF domain PEEM images; filled symbols for different temperature runs. Open triangles show temperature dependence of magnetic superstructure reflections in LEED [23]; stars show neutron scattering data [4]. 


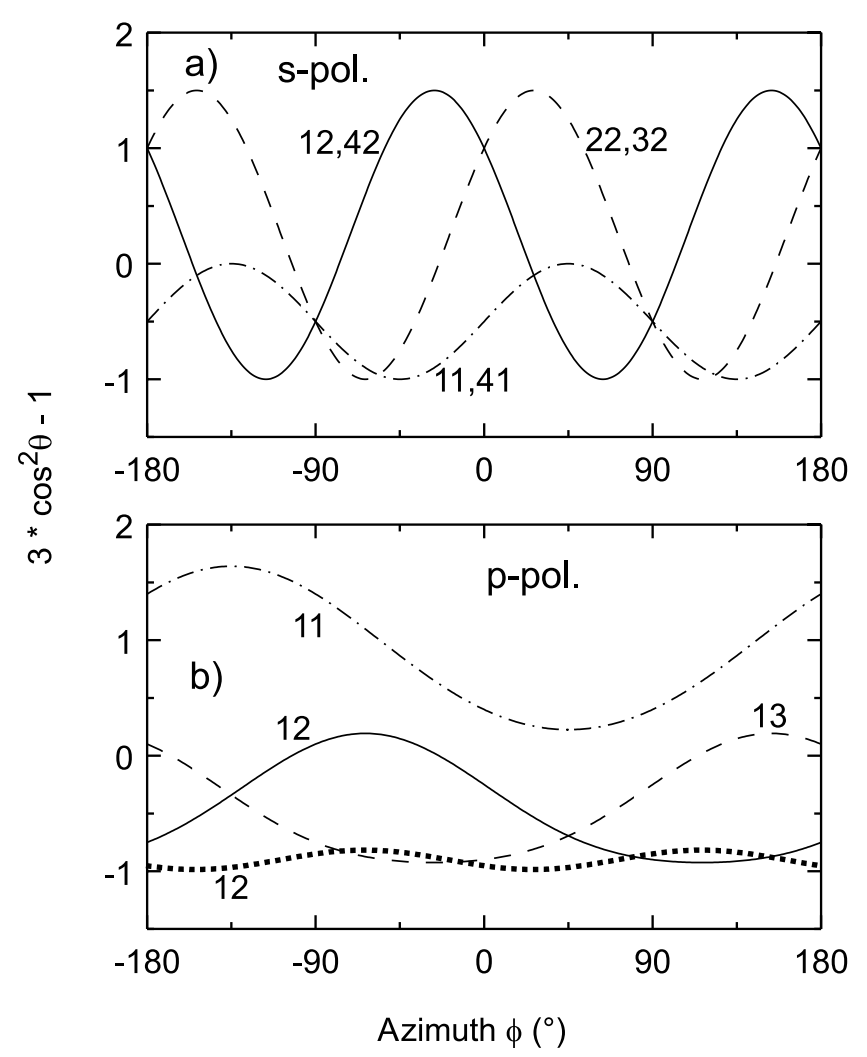

FIG. 3. Expected magnitude of the angle dependent signal in the absorption spectrum for different $\mathrm{AF}$ domains of $\mathrm{NiO}$ as a function of azimuthal crystal orientation (measured by $\phi$ ) for a (100) surface. The angle dependent part varies as a $3 \cos ^{2} \theta-$ 1 , where $\theta$ is the angle between electric field and magnetic moment. $0^{\circ}$ corresponds to a [001] direction coinciding with the plane of incidence. The numbers $i j$ refer to the domains given in Table I, the first for the $T$ domain and the second for the $S$ domain. (a) $s$-polarized excitation (curves for the other domains are shifted by $90^{\circ}$ with respect to those shown); (b) $p$-polarized excitation (the curve for $T_{42}$ is shifted by $180^{\circ}$ from $T_{12}$ ), for bulk termination; dotted line labeled 12 is for out-of-plane component set to zero.

occurs at the positions halfway between the zero contrast positions, and has the same magnitude both ways. The line is a $\sin (a \phi)$ curve, where $a=2$ is the best fit.

Figure $4 \mathrm{~b}$ shows the variation of the branching ratio of the two structures of the $\mathrm{Ni} 2 p_{1 / 2}$ absorption spectrum for individual domains. These curves represent the magnitude of the angular dependent part in the absorption spectrum, and can therefore be directly compared to the model curves shown in Fig. 3. The separation of the peaks and their location with respect to the crystal directions can be used to identify the domains present. The 11/41 and 21/31 domains with dominating component perpendicular to the surface should show maxima at $+45^{\circ}$ from an in-plane [01] axis (dot-dashed line in Fig. 3a), i.e., a peak separation of $90^{\circ}$. This is in contrast to the experimental result of $(63 \pm 3)^{\circ}$ peak separation. The result suggests that the contrast is caused by the $12 / 42$ and $22 / 32$ domains, for which $54^{\circ}$ are expected between the peaks. The remaining difference may be caused by admixture of other domains with large in-plane components, i.e., $T_{13} / T_{43}$ and

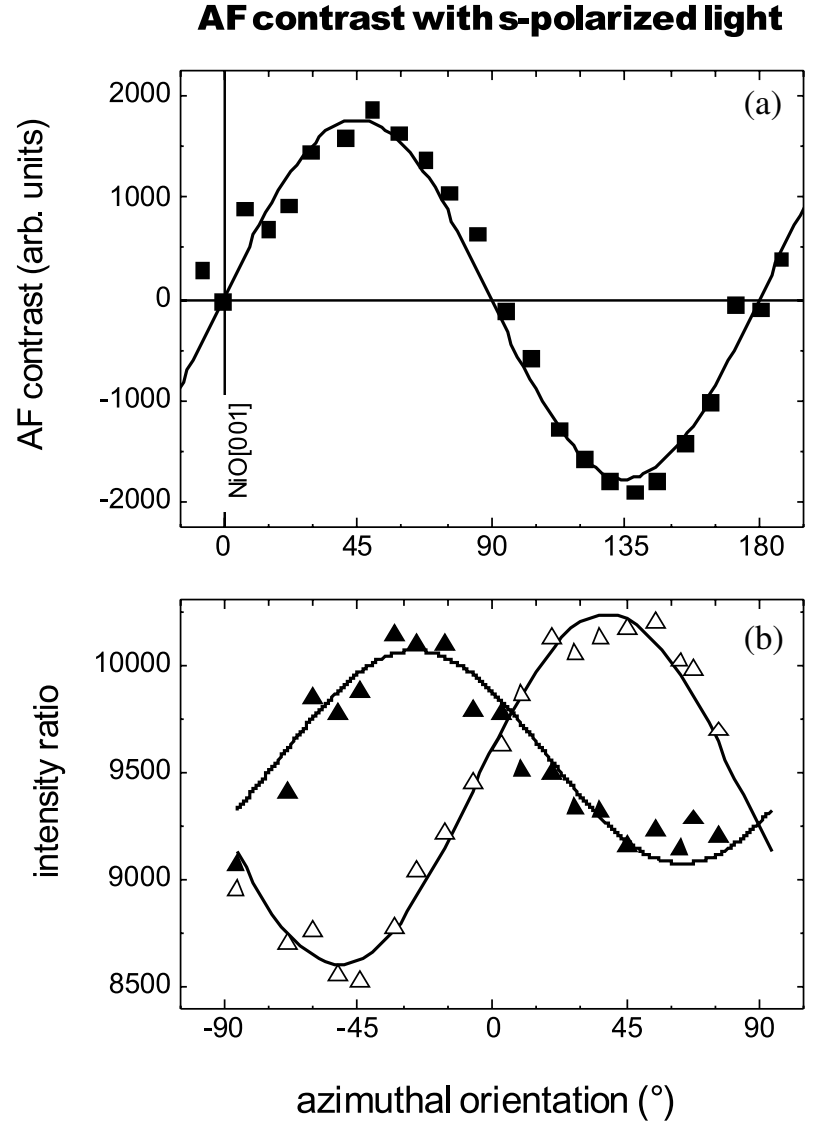

FIG. 4. Polarization dependence of AF domain contrast for $\mathrm{NiO}(100)$. (a) Measured variation of antiferromagnetic domain contrast (solid squares) with azimuthal sample orientation for $s$-polarized light. $0^{\circ}$ corresponds to a [001] direction coinciding with the plane of incidence. The data show expected angular dependence for domains $T_{i 2} / T_{i 3}$. (b) Change of chromatic contrast, $I(871 \mathrm{eV}) / I(872 \mathrm{eV})$, for $s$ polarization, representing the magnitude of the angle dependent part in the absorption spectrum, averaged separately over bright (empty triangle) or dark (filled triangle) domains as a function of azimuthal orientation [different surface from (a)].

$T_{23} / T_{33}$, respectively. The mean branching ratio (averaged over both domains) changes between $0^{\circ}$ and $+90^{\circ}$, and the maximum in one curve does not coincide with the minimum of the other. This demonstrates that the angle between the magnetic moments projected on the surface is not $90^{\circ}$, since then the mean branching ratio should be identical at all positions where the contrast disappears. These findings confirm our assignment of the experimental contrast to domains with large in-plane components of the magnetic moments.

The exchange bias is known to decrease with increased roughness of the antiferromagnetic surface [24]. To address this problem, we studied the $\mathrm{NiO}$ roughened by sputtering. With $s$ polarized light we still observe AF domain contrast with a similar angular dependence as discussed above, although the contrast is reduced in comparison to cleaved surfaces. However, there was no domain contrast observable with $p$ polarized light. Figure $3 b$ shows the variation of the angular dependent term for $T_{1 j}$ domains 
for $p$-polarized light, assuming bulk termination. The well separated curves for $T_{i 1}$ and $T_{i 2, i 3}$ indicate that these domains should always be distinguishable. The null contrast shows that the out-of-plane components of the magnetic moments are quenched for the domains present on the surface: in this case the magnetic moments are at angles between $90^{\circ}$ and $75^{\circ}$ to the electric field, depending on the azimuth. The remaining contrast of only $6 \%$ of the maximum is below our detection limit. The quenching of the perpendicular components of the magnetic moments indicates a magnetic surface relaxation of the antiferromagnetic $\mathrm{NiO}$ surface.

The size of the domains observed here is much larger than was concluded from measurements on thin films [17]. The reappearance of an unchanged domain pattern after heating above the Néel temperature shows that the domain topography is determined by defects built into the material during growth. Therefore, the growth of a thin film with a large number of nucleation sites will lead to quite different domain patterns. The apparent variation in domain size for different surfaces is relevant for the exchange bias phenomenon, since for a compensated AF surface the domain walls which have uncompensated spins are thought to be the source of the bias $[25,26]$.

We have analyzed the magnetic moment orientations at the surface of antiferromagnetic $\mathrm{NiO}$ by spatially resolved polarization dependent x-ray absorption spectroscopy monitored by total electron yield in a photoemission microscope. By utilizing complete control over light polarization offered by a state-of-art synchrotron radiation beam line, a full characterization of AF surfaces is possible, i.e., all 12 domain types possible for bulk $\mathrm{NiO}$ can be distinguished. On (100) surfaces of bulk NiO, stripe-shaped $T$ domains up to a few tens of $\mu \mathrm{m}$ wide are observed, with boundaries running along [001] directions in the surface. For cleaved surfaces, the angular dependence of the AF contrast shows a bulk termination of the magnetic structure. For the examples studied we conclude from the detailed polarization dependence that primarily $S$ domains with large in-plane components of the magnetic moment are present. The domain topology is not affected by macroscopic defects on the surface, and reappears unchanged after heating above the Néel temperature. These findings indicate that the surface domains are governed by bulk domains terminating at the surface. For surfaces with a higher degree of roughness, $s$-polarized excitation reveals an essentially unaffected domain pattern, but no domain contrast is observed with obliquely incident $p$-polarized light, indicating a magnetically relaxed surface.

We thank V. M. Uzdin for informative discussions, and E. Kisker for his support. This work was supported by BMBF under Grant No. 05 SL8 PFA 5.
*Present address: Max Planck Institut für Mikrostrukturphysik, Weinberg 2, 06120 Halle, Germany.

[1] G. A. Prinz, Science 282, 1660 (1998).

[2] J. Nogués and I. K. Schuller, J. Magn. Magn. Mater. 192, 203 (1999).

[3] C. Shull, W. Strausser, and E. Wollan, Phys. Rev. 83, 333 (1951).

[4] W. Roth, Phys. Rev. 111, 772 (1958).

[5] W. L. Roth, J. Appl. Phys. 31, 2000 (1960).

[6] T. Yamada, S. Saito, and Y. Shimomura, J. Phys. Soc. Jpn. 21, 672 (1966).

[7] T. Yamada, J. Phys. Soc. Jpn. 21, 650 (1966).

[8] J. Baruchel, M. Schlenker, K. Kurosawa, and S. Sito, Philos. Mag. B 43, 853 (1981).

[9] H. Komatsu and M. Ishigame, J. Mater. Sci. 20, 4027 (1985).

[10] A. Swan, M. Marynowski, W. Franzen, M. El-Batanouny, and K. Martini, Phys. Rev. Lett. 71, 1250 (1993).

[11] M. Marynowski, W. Franzen, M. El-Batanouny, and V. Staemmler, Phys. Rev. B 60, 6053 (1999).

[12] R. Wiesendanger, H.-J. Güntherodt, G. Güntherodt, R. Gambino, and R. Ruf, Phys. Rev. Lett. 65, 247 (1990).

[13] H. Hopster, Phys. Rev. Lett. 83, 1227 (1999).

[14] B. T. Thole, G. van der Laan, and G. A. Sawatzky, Phys. Rev. Lett. 55, 2086 (1985).

[15] D. Alders, J. Vogel, C. Levelut, S. D. Peacor, T. Hibma, M. Sacchi, L. H. Tjeng, C. T. Chen, G. van der Laan, B. T. Thole, and G. A. Sawatzky, Europhys. Lett. 32, 259 (1995).

[16] D. Spanke, V. Solinus, D. Knabben, F. U. Hillebrecht, F. Ciccacci, L. Gregoratti, and M. Marsi, Phys. Rev. B 58, 5201 (1998).

[17] J. Stöhr, A. Scholl, T. J. Regan, S. Anders, J. Lüning, M. R. Scheinfein, H. A. Padmore, and R. L. White, Phys. Rev. Lett. 83, 1862 (1999).

[18] W. Gudat and C. Kunz, Phys. Rev. Lett. 29, 169 (1972).

[19] PEEM 350 by Staib Instruments; see also W. Engel, M.E. Kordesch, H. H. Rothermund, S. Kubala, and A. v. Oertzen, Ultramicroscopy 36, 16 (1993).

[20] K. Sawhney, F. Senf, M. Scheer, F. Schäfer, J. Bahrdt, A. Gaupp, and W. Gudat, Nucl. Instrum. Methods Phys. Res., Sect. A 390, 395 (1997).

[21] B. Fromme, C. Koch, R. Deussen, and E. Kisker, Phys. Rev. Lett. 75, 693 (1995).

[22] The grey scale of the image has 256 levels, which have been determined by $255\left(A-A_{\min }\right) /\left(A_{\max }-A_{\min }\right) . A_{\min }$ and $A_{\max }$ are the maximum and minimum of the asymmetries of the image. Alders et al. [15] use the intensity ratio $Q$ of absorption coefficients (as measured by total yield) at 871.2 and $872.2 \mathrm{eV}: A=(Q-1) /(Q+1)$.

[23] T. Wolfram, R. DeWames, W. Hall, and P. Palmberg, Surf. Sci. 28, 45 (1971).

[24] J. Nogues, D. Lederman, T. Moran, I. Schuller, and K. Rao, Appl. Phys. Lett. 68, 3168 (1996).

[25] P. Miltényi, M. Gierlings, J. Keller, B. Beschoten, G. Güntherodt, U. Nowak, and K. D. Usadel, Phys. Rev. Lett. 84, 4224 (2000).

[26] H. Matsuyama, C. Haginoya, and K. Koike, Phys. Rev. Lett. 85, 646 (2000). 